\title{
Proctitis ulcerosa activa y refractaria: Una puesta al día
}

\author{
GINO CASELLI M. ${ }^{1}$, GEORGE PINEDO M. ${ }^{1}$, \\ ÁlVARO ZÚÑIGA D. ${ }^{1}$, MANUEL ÁLVAREZ L. ${ }^{2}$
}

\section{Active and refractory ulcerative proctitis: An update}

Ulcerative colitis (UC) is a chronic inflammatory disease of unknown etiology that affects a variable length of the colon, starting from the rectum. When the disease is confined to the rectum is called ulcerative proctitis (UP). Several studies have unsuccessfully attempted to determine the factors that determine the extent of involvement. The goals of therapy in UP are to induce and maintain remission of symptoms and disease. Topical treatment with 5-aminosalicylates (5-ASA) is the treatment of choice to induce remission. In the maintenance phase, long-term follow up studies suggest that treatment with 5-ASA is better than placebo, to maintain the disease inactive. For those patients that do not respond to treatment with topical 5-ASA or have a moderate to severe disease, there are additional therapies such as oral 5-ASA, topical or systemic corticosteroids, immunomodulators, biological therapies (Infliximab) and cyclosporine. Surgery is seldom needed.

(Rev Med Chile 2010; 138: 109-116).

Key words: Colitis, ulcerative, meselamine, proctitis.

'Unidad de Cirugía Colorrectal y ${ }^{2}$ Departamentos de Cirugía Digestiva y Gastroenterología, Hospital Clínico, Pontificia Universidad Católica de Chile.

Recibido el 5 de marzo de 2009, aceptado el 1 de diciembre de 2009.

Correspondencia a: Dr. George Pinedo Mancilla E-mail: gpinedo@med.puc.cl Unidad de Cirugía Colorrectal. Departamento de Cirugía

Digestiva.

Pontificia Universidad Católica de Chile

Marcoleta 367. Santiago, Chile Teléfono: $+56-2-3548283$ Fax: $+56-2-6382793$
L a colitis ulcerosa (CU) es una enfermedad inflamatoria crónica del tracto digestivo, de causa desconocida. Esta enfermedad se caracteriza por un curso crónico con alternancia de períodos de actividad con períodos de remisión clínica. La incidencia de CU en Norteamérica presenta un rango que va entre 2,2 a 14,3 por cada 100.000 habitantes al año ${ }^{1}$. El compromiso contínuo desde el recto hacia proximal presenta una extensión variable entre pacientes y en el curso de la enfermedad ${ }^{2}$. La importancia de la extensión radica en que determina la presentación clínica, tratamiento, curso y pronóstico de la $\mathrm{CU}^{2}$. Según la extensión, se clasifican en rectitis o proctitis ulcerosa (PU) cuando esta afecta sólo al recto, colitis izquierda o distal cuando la inflamación es distal al ángulo esplénico y colitis extensa cuando la inflamación llega hasta el colon transverso, derecho o tiene una afectación de todo el colon ${ }^{2}$. Varios estudios han intentado establecer los factores que de- terminarían la extensión de la afectación. Entre los factores asociados a una extensión exclusivamente rectal están pacientes en edad media, ausencia de hemorragia grave y ausencia de manifestaciones extraintestinales ${ }^{3}$. Sin embargo, estos factores no han sido replicados, por lo que hasta la actualidad no hay factores sólidamente asociados a $\mathrm{PU}^{1,4}$. La mayoría de los nuevos casos diagnosticados en adultos se presentan como una PU o una colitis distal ${ }^{1,5}$. Aunque comunicaciones iniciales señalaban que la PU tenía una baja frecuencia ${ }^{6}$, estudios recientes informan que la incidencia de PU ha ido aumentando hasta $48 \%$ y $60 \% \%^{4,7}$ considerando que la incidencia de la colitis ha ido disminuyendo en la mayoría de las áreas geográficas ${ }^{8}$.

Algunos autores han sugerido que la PU representa una entidad clínica completamente distinta a la $\mathrm{CU}^{8,9}$. Sin embargo, la mayoría concuerda que la PU representa una forma acotada o inicial de la CU con el potencial de extenderse hacia los segmentos 
proximales del colon ${ }^{10-12}$. La PU, a diferencia de las otras formas de presentación de CU más extensas, se caracteriza clínicamente por sangrado y/o pujo rectal, sin síntomas sistémicos ni alteración en el examen físico o en los exámenes de laboratorio y puede ser tratada exclusivamente con terapias tópicas por vía rectal ${ }^{7}$.

Los 5-aminosalicilatos (5-ASA) y los corticoides están disponibles para uso tópico como agentes antiinflamatorios locales ${ }^{7}$. Estos fármacos pueden llegar directamente al sitio de la inflamación, lo que disminuye la absorción sistémica y minimizan los potenciales efectos secundarios ${ }^{7,13}$. Los 5-ASA orales también son una alternativa previa al uso de corticoides sistémicos, ya que estos últimos presentan diversos riesgos y limitaciones en su uso a corto y largo plazo y se reservan para las PU activas muy intensas ${ }^{14}$. Cuando existe refractariedad a los tratamientos anteriores, los fármacos que pueden ser utilizados para inducir y mantener la remisión son los inmunomoduladores como la azatioprina ${ }^{15}$, la 6 -mercaptopurina ${ }^{16}$ y la terapia con Infliximab ${ }^{17,18}$.

La mortalidad y el riesgo de cáncer asociado no es mayor que la población general ${ }^{2}$ por lo que usualmente la PU ha sido considerada una forma de presentación leve de la CU. Sin embargo, cerca de $23 \%$ de los pacientes portadores de PU puede llegar a una colectomía ${ }^{19}$ y entre 41 y $54 \%$ de los pacientes aumentará su extensión después de 10 años de enfermedad ${ }^{2}, 11$, lo que indica que la PU no siempre constituye una enfermedad leve.

Esta revisión tiene por objetivo discutir el tratamiento actual y entregar nuevos conceptos sobre la PU activa y refractaria.

\section{Preparados 5-ASA}

El tratamiento tópico ha sido utilizado por largo tiempo y ha ofrecido la ventaja de entregar una alta dosis del compuesto directamente al sitio de inflamación, minimizando la absorción y por lo tanto limitando la frecuencia de efectos adversos sistémicos ${ }^{20}$. Las preparaciones administradas por vía rectal de 5-ASA son el tratamiento de elección de la PU y CU distal de intensidad leve y moderada. Varias formas de este compuesto han sido probadas en distintos estudios clínicos, incluyendo supositorios, enemas, espumas y gel. Los supositorios de mesalazina administrados a dosis de $1 \mathrm{~g} /$ día, de preferencia en la noche, deberían ser considerados como el tratamiento de elección en la PU activa, ya que son superiores a 5-ASA orales con $91 \%$ versus $41 \%$ en inducción de la remisión ${ }^{5,11,21-24}$. Estudios con cintigrafía han demostrado que esta droga llega de forma consistente al recto y al sigmoides distal hasta una extensión de $18-20 \mathrm{~cm}$ desde el margen anal ${ }^{25}$, siendo igual de efectivo que los enemas, pero mejor tolerados y preferidos por los pacientes ${ }^{26}$. La dosificación de los enemas de 5-ASA es de $4 \mathrm{~g}$ en $60 \mathrm{ml}$ con una posología de 1-2 veces al día por 4 semanas. Aunque igualmente efectivos que el enema, las espumas y el gel podrían ofrecer la ventaja de una retención intraluminal más prolongada, distribución más homogénea en la mucosa inflamada y mejor tolerancia por los pacientes ${ }^{26,28}$. El tratamiento de mantención está indicado para todos los casos y la duración mínima es de $1 \mathrm{anno}^{29,30}$, existiendo un pequeño porcentaje de pacientes que podría suspender la terapia completamente, pero con recaídas cercanas a un $86 \%$ al completar doce meses de tratamiento ${ }^{31}$. El algoritmo del manejo de PU activa y remisión se muestra en la Figura 1.

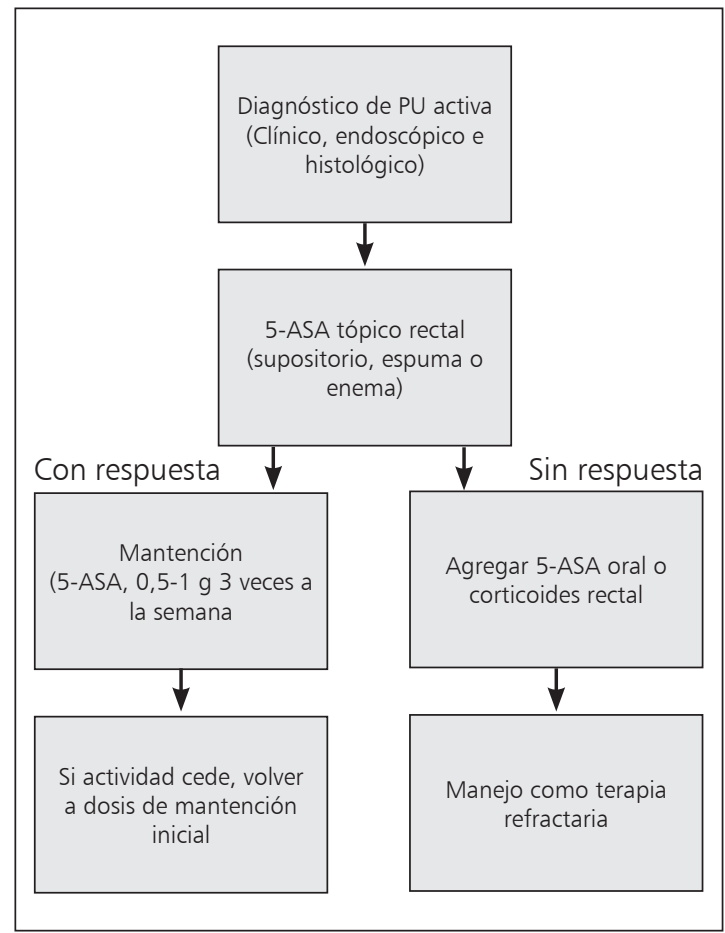

Figura 1. Algoritmo de tratamiento de PU activa y mantención de remisión. Adaptado de Clinical Guidelines. Inflamm Bowel Dis 2006; 12: 972-8. Regueiro et al ${ }^{1}$. 


\section{5-ASA de administración oral}

Cuando el paciente rehusa el uso de tratamiento tópico o si después de 2-4 semanas de tratamiento con 5-ASA por vía rectal no hay respuesta, los 5-ASA orales (mesalazina o sulfasalazina) son una alternativa. Estudios clínicos randomizados han demostrado beneficios en la adición de compuestos 5-ASA orales (mesalazina) con una dosis superior a los $3 \mathrm{~g} /$ día en la CU distal activa $^{32}$. No hay estudios adecuados que demuestren la eficacia de estos compuestos orales como monoterapia para la PU, aunque su eficacia sí ha sido demostrada para CU más extensas. Se ha postulado que sería especialmente útil para evitar extensión proximal de la enfermedad en la fase de mantención del tratamiento ${ }^{32,33}$. Su administración puede asociarse a fenómenos alérgicos y cefalea entre otros efectos secundarios ${ }^{13}$.

\section{Los corticoesteroides en el tratamiento de la PU}

Para aquellos pacientes en que no hay respuesta después de 2-4 semanas de tratamiento tópico con aminosalicilatos se considera el uso de corticoesteroides. Varios corticoides han sido eficazmente administrados por vía rectal (supositorios, enemas, espumas), incluyendo hidrocortisona (100 a 200 $\mathrm{mg}$ en 60 a $200 \mathrm{ml}$ ), betametasona, prednisolona fosfato $^{34} \mathrm{y}$ últimamente la inclusión como agente terapéutico de la budesonida ${ }^{35}$. Los enemas de hidrocortisona fueron significativamente superiores al placebo con $55 \%$ versus $10 \%$ en inducción de remisión ${ }^{36}$ y tienen una eficacia comparable a los corticoides sistémicos y con menor inhibición del eje hipotálamo-hipofisiario-adrenal, ya que existe directa absorción a través de las venas rectales superiores y media a la circulación sistémica sin paso por el sistema portal hepático ${ }^{35}$. La budesonida ha sido largamente estudiada durante la última década, al compararla con mesalazina y otros glucocorticoides. Un estudio comparó la respuesta de la espuma de budesonida y espuma de hidrocortisona en pacientes que fallaron con mesalazina, logrando $52 \%$ de respuesta en el grupo de budesonida y $37 \%$ de remisión en el grupo que utilizó espuma de hidrocortisona ${ }^{36}$. La remisión con la espuma de budesonida se lograría en 19\% con una dosificación mínima de $2 \mathrm{mg} / 100 \mathrm{ml}$ por un período de al menos 6 semanas, aunque dosis de $8 \mathrm{mg}$ en $100 \mathrm{ml}$ lograrían remisión en un porcentaje más elevado de $27 \%$ versus $4 \%$ del placebo ${ }^{35-37}$. No hay estudios que avalen el uso de corticoides para la mantención de la remisión de la $\mathrm{PU}^{1}$.

A pesar de los beneficios entregados por los compuestos 5-ASA y corticoides de administración rectal, algunos pacientes fallan en lograr la remisión y requieren de terapia adicional. El incumplimiento de la administración de medicamentos es considerado como el factor de refractariedad más importante de la $\mathrm{PU}$, por lo que al reintroducir la terapia se podría lograr una buena respuesta. Los pacientes deben además ser evaluados para descartar hipersensibilidad a los aminosalicilatos (colitis alérgica), caracterizado por dolor abdominal y diarrea ${ }^{1}$ y mediante exámenes o endoscopía descartar una sobreinfección por Clostridium difficile, una extensión de la enfermedad hacia segmentos proximales del colon o la presencia de una enfermedad de Crohn. La infección por Citomegalovirus también debe ser excluida en los pacientes refractarios. Los pacientes que no responden a terapia rectal y/u oral (5-ASA y corticoesteroides) representan un importante problema; aquí las opciones incluyen azatioprina (AZA), 6-mercaptopurina (6-MP), inmunomoduladores, Infliximab, antibioticoterapia e incluso cirugía ${ }^{1}$.

\section{Antibioticoterapia}

A diferencia de la enfermedad de Crohn, no se ha probado la efectividad de los antibióticos en la colitis ulcerosa ${ }^{1}$. Tanto la ciprofloxacina, tobramicina y metronidazol han sido estudiados y a pesar de una clara tendencia a la mejoría, no mostraron superioridad en cuanto a la inducción o mantención de la remisión en $\mathrm{CU}^{38}$. A pesar que en algunos estudios hubo una respuesta clínica y endoscópica cercana al $80 \%$, la remisión no fue sostenida y la recurrencia fue similar al placebo ${ }^{39}$. No hay estudios del efecto de los antibióticos en PU.

\section{Azatioprina y 6-mercaptopurina en PU}

La AZA y la 6-MP representan una pieza clave en la terapia de las EII ${ }^{40}$, fundamentalmente en los casos corticodependientes y resistentes. Evidencia científica sólida disponible sobre el papel de la AZA y 6-MP en la CU es más limitada que en la $\mathrm{EC}^{41}$; se han publicado 3 estudios controlados con placebo que evalúan la eficacia de estos fármacos en la inducción de remisión de pacientes con CU activa, en los cuales se ha determinado que AZA no 
tiene efectos en lograr la remisión, pero sí disminuyendo la proporción de recidiva ${ }^{42,43}$. Tampoco ha sido mayormente evaluada en cuanto a la mantención de la remisión de la CU. No existen estudios que evalúen la efectividad de estos compuestos exclusivamente en PU o colitis distal, pero es una alternativa válida en el tratamiento ${ }^{32}$.

\section{Ciclosporina}

Existen datos en la CU grave que demuestran que la ciclosporina es eficaz en inducir remisión ${ }^{44}$. La respuesta relativamente rápida hace que el uso de ciclosporina sea potencialmente atractivo, pero el beneficio a largo plazo no es claro, sobretodo cuando efectos adversos potencialmente fatales pueden aparecer, como la nefrotoxicidad y las infecciones oportunistas. No existen estudios clínicos randomizados que muestren efectividad de este inmunosupresor para la PU refractaria y los estudios son sólo anecdóticos ${ }^{45,46}$.

\section{Terapia biológica en la PU}

Las terapias biológicas intentan recuperar el balance entre efectos proinflamatorios y antiinflamatorios que se observa en las EII. El Infliximab es un anticuerpo monoclonal quimérico (IgG) derivado de un $\mathrm{ADN}$ recombinante, formado por genes de origen humano y murino. Se une y neutraliza al factor de necrosis tumoral de tipo alfa $(\mathrm{TNF} \alpha)$, logrando interrumpir la cascada secuencial de activación de las vías inflamatorias mediadas por esta citoquina. El uso de Infliximab se revisó en los estudios randomizados y controlados ACT 1 y ACT 2, demostrando su beneficio en pacientes con CU moderada y severamente activa, además de la CU refractaria ${ }^{47}$. Estos ensayos, sin embargo, excluyeron en la práctica a los pacientes con sólo PU. Dentro de las ventajas de este fármaco se incluyen un rápido inicio de acción y la posibilidad de conseguir la normalización endoscópica e histológica de la mucosa. Se demostró 30\% de remisión y $60 \%$ de mejoría en los pacientes. Cifras cercanas a $70 \%$ de los pacientes refractarios a 5-ASA, corticoides o AZA/6-MP responden a Infliximab ${ }^{48}$. Sin embargo, el uso de Infliximab en esta condición es escaso ${ }^{1}$. El algoritmo del manejo de PU refractaria se muestra en la Figura 2.

\section{Otras terapias de investigación}

Parches transdérmicos y enemas de nicotina ${ }^{47}$, heparinas de bajo peso molecular ${ }^{50}$, ácidos grasos de cadena liviana de administración $\operatorname{rectal}^{45} y$ probióticos ${ }^{51}$ han sido descartados para el manejo de la CU izquierda o PU, dado su bajo nivel de evidencia y su baja eficacia en la inducción de la remisión de la crisis activa.

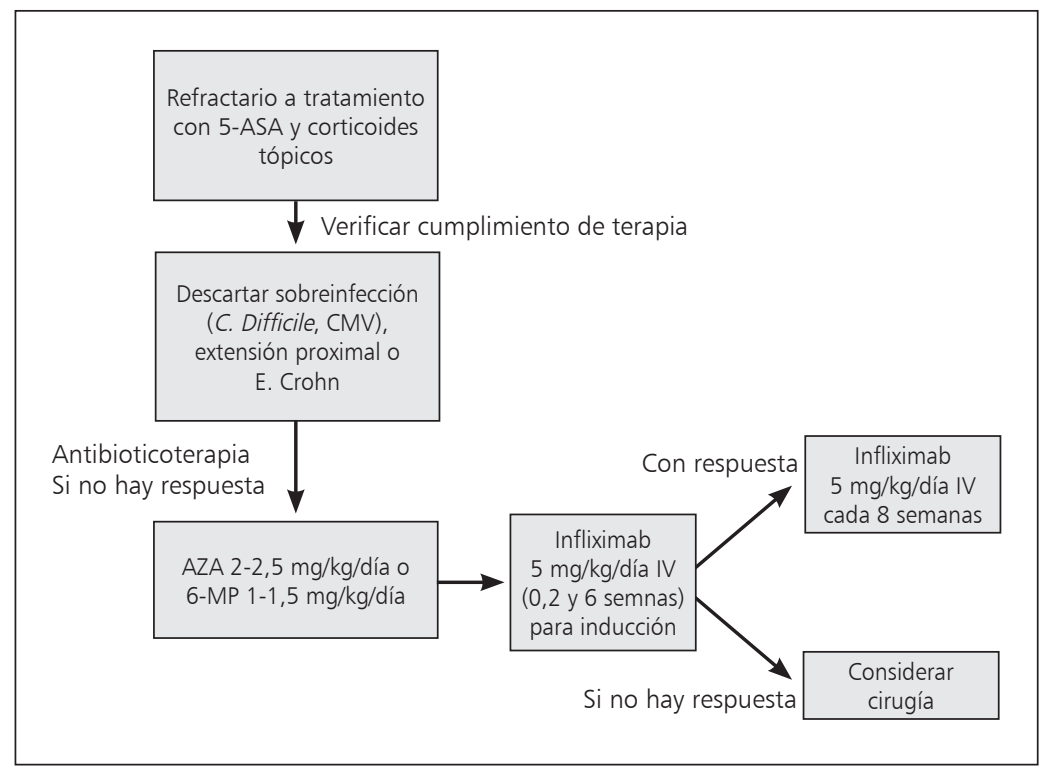
miento de PU refractaria a 5-ASA y corticoides. 


\section{Cirugía en la proctitis ulcerosa}

A pesar que la PU puede ser de difícil manejo $y$ a veces refractario a toda terapia, es infrecuente que la cirugía sea considerada como una opción de tratamiento. Las cifras van entre 2-9\% a 5 años ${ }^{52,53}$ hasta $23 \%$ a 20 años $^{54}$. Para aquellos pacientes que sí requerirán cirugía, la proctocolectomía con ileostomía terminal o la confección de un reservorio ileal con anastomosis reservorio-anal constituyen las opciones ${ }^{1}$. Destacamos que no hay estudios publicados que demuestren resultados a corto o largo plazo de la cirugía en PU refractaria.

\section{¿Cual es el futuro del tratamiento de la PU?}

Los protocolos de estrategia terapéutica basados en la mejor evidencia científica hacen que el pronóstico de los pacientes con PU haya mejorado de forma importante durante los últimos años. Con la disponibilidad de las terapias biológicas y los avances en cirugía colorrectal se abren nuevas expectativas para evaluar la utilidad de estas alternativas en las PU refractarias al tratamiento clásico. Esto ha estado asociado a la aparición de nuevas drogas con las cuales se obtendría una mejoría de los síntomas con menos efectos adversos, los que no están aún disponibles.

El uso de enemas de factor de crecimiento epidermal (EGF) ha entregado buenos resultados y un tratamiento efectivo en la colitis izquierda y PU leve a moderada cuando ha sido asociada a mesalamina oral ${ }^{55}$. Este compuesto estimula la migración y proliferación celular y repara las injurias de la mucosa gástrica, intestinal y colónica. Estudios muestran una remisión de $83 \%$ en el grupo del enema de EGF vs $8 \%$ del grupo placebo después de 2 semanas de tratamiento. A pesar de reducir la actividad y de inducir remisión clínica, se deben realizar estudios clínicos que comparen $E G F$ versus dosis altas de mesalamina o corticoides ${ }^{55}$.

El Rebamipide es un aminoácido derivado de la $2(1 \mathrm{H})$-quinolinona, que fue utilizado para la protección de la mucosa gástrica y duodenal. Éste actúa a través de la supresión de las funciones de los neutrófilos, la estimulación de la regeneración de células epiteliales y un aumento de la expresión del factor de crecimiento epidérmico (EGF) y de su receptor. A través de un estudio prospectivo se determinó que el enema de Rebamipide en colitis distal y PU activa como terapia local tópica podía ser efectivo en el tratamiento de la enfermedad activa leve y moderada ${ }^{56}$. No se presentaron efectos adversos relacionados con el Rebamipide en los 16 pacientes incluidos en el estudio ${ }^{57}$.

El Tacrolimus es un fármaco inmunosupresor producido por el Streptomyces tsukubaensiscon, que ha sido empleado principalmente en trasplantes y en enfermedades autoinmunes. Posee un mecanismo similar al de la ciclosporina, pero es mejor tolerado y tiene una potencia 100 veces mayor que ésta. Actúa directamente sobre los linfocitos T, inhibiendo la transcripción de la IL2, disminuye la respuesta de los linfocitos a los antígenos $^{58}$ e inhibe la liberación de mediadores de la inflamación desde los mastocitos y basófilos. Lawrence et $\mathrm{al}^{58}$, lograron una remisión completa después de 8 semanas de tratamiento con Tacrolimus rectal en el 75\% de los pacientes estudiados, todos ellos resistentes a terapia convencional. Los pacientes que no respondieron presentaron progresión hacia enfermedad proximal, donde el compuesto no llegó de forma tópica. Ensayos clínicos randomizados son necesarios para evaluar su efectividad versus placebo.

En resumen, la administración tópica de 5-ASA en supositorios, enema o espumas es el tratamiento de elección para la mayoría de los pacientes con PU. La administración local de 5-ASA es más eficaz que los 5-ASA orales, pero la combinación oral y tópica debe ser considerada para los casos sin respuesta adecuada a alguna de estas terapias por separado. Corticoides tópicos son el tratamiento de segunda línea ya sea como tratamiento único o en combinación con 5-ASA tópico. El tratamiento de mantención está indicado en todos los casos y el uso preferente corresponde a los 5-ASA tópicos, siendo la formulación oral también una alternativa. Los pacientes refractarios o intolerantes a 5-ASA pueden requerir inmunomoduladores o terapia biológica. Los esteroides sistémicos o la cirugía debe ser empleada en casos muy especiales.

\section{Referencias}

1. Regueiro M, Loftus E, Steinhart H, Cohen R. Clinical Guidelines for the medical management of left-sided ulcerative colitis and ulcerative proctitis: Summary statement. Inflamm Bowel Dis 2006; 12: 972-8.

2. Silverberg M, Satsangi J, Ahmad T, Arnott I, Bernstein C, Brant S, et al. Toward an integrated clinical, molecular and serological classification of inflammatory bowel disease: Report of a Working Party of the 2005 Montreal 
World Congress of Gastroenterology. Canad J Gastroenterol 2005; 19 Suppl A: 5-36.

3. Farmer R, Easley K, Rankin G. Clinical patterns, natural history, and progression of ulcerative colitis. A longterm follow-up of 1116 patients. Dig Dis Sci 1993; 38: 1137-46.

4. Langholz E, Munkholm P, Davidsen M, Bonder V. Course of ulcerative colitis: analysis of changes in disease activity over years. Gastroenterology 1994; 107: 3-11.

5. Loftus E. Clinical epidemiology of inflammatory bowel disease: incidence, prevalence and environmental influences. Gastroenterology 2004; 126: 1504-17.

6. Powell-Tuck J, Ritchie J, Lennard-Jones J. The prognosis of idiopathic proctitis. Scand J Gastroenterol 1977; 12: 727-32.

7. Pica R, Paoluzi O, Iacopini F, Marcheggiano A, Crispino P, Rivera M, et al. Oral mesalazine (5-ASA) treatment may protect against proximal extention of mucosal inflammation in ulcerative proctitis. Inflamm Bowel Dis 2004; 10: 731-6.

8. Russel M, Stockbrugger R. Epidemiology of inflammatory bowel disease: An update. Scand J Gastroenterol 1996; 31: 417-27.

9. Farmer R. Nonspecific ulcerative proctitis. Gastroenterol Clin North Am 1987; 16: 157-74.

10. Ekbom A, Helmick C, Zack M, Adami H. Ulcerative proctitis in central Sweden 1965-1983: A populationbased epidemiological study. Dig Dis Sci 1991; 36: $97-$ 102.

11. Campieri M, De Franchis R, Bianchi Porro G, Ranzi T, Brunetti G, Barbara L. Mesalazine (5-Aminosalicylic acid) suppositories in the treatment of ulcerative proctitis or distal proctosigmoiditis: A randomized controlled trial. Scand J Gastroenterol 1990; 25: 663-8.

12. Breschi G, Parisi G, Gambardella L, Banti S, Bertoni M, Rindi G, et al. Evaluation of clinical patterns in ulcerative colitis: a long-term follow-up. Int J Clin Pharmacol Res 1997; 17: 17-22.

13. Loftus E JR, Kane S, Bjorkman D. Systematic review: short-term adverse effects of 5-aminosalicylic acid agents in the treatment of ulcerative colitis. Aliment Pharmacol Ther 2004; 19: 179-89.

14. Truelove S, Jewell D. Intensive intravenous regimen for severe attacks of ulcerative colitis. Lancet 1974; 1: 10418.

15. Hawthorne A, Logan R, Hawkey C, Foster P, Axon A, Swarbrick E, et al. Randomized controlled trial of azathioprine withdrawal in ulcerative colitis. BMJ 1992; 305: 20-2.

16. George J, Present DH, Pou R Bodian C, Rubin P. The long-term outcome of ulcerative colitis treated with 6-mercaptopurine. Am J Gastroenterol 1996; 91: 1711-4.

17. Eaden J, Abrams K, Mayberry J. The risk of colorrectal cancer in ulcerative colitis: a meta-analysis. Gut 2001; 48: 526-35.

18. Rutgeerts P, Sandborn W, Feagan B, Reinisch W, Olson A, Johanns J, et al. Infliximab for induction and maintenance therapy for ulcerative colitis. N Engl J Med 2005; 353: 2462-76.

19. Mir-Madjlessi S, Michener W, Farmer R. Course and prognosis of idiopathic ulcerative proctosigmoiditis in young patients. J Pediatr Gastroenterol Nutr 1986; 5: 571-5.

20. Gionchetti P, Rizzello F, Morselli C, Campieri M. Review article: Problematic proctitis and distal colitis. Aliment Pharmacol Ther 2004; 20 (Suppl 4): 93S-96S.

21. Gionchetti P, Rizzello F, Belluzzi A. 5-Aminosalicylic acid as enemas or suppositories in distal ulcerative colitis. J Clin Gastroenterol 1988; 10: 406-9.

22. Gionchetti P, Rizzello F, Venturi A, Ferretti M, Brignola C, Miglioli M, et al. Comparison of oral with rectal mesalazine in the treatment of ulcerative proctitis. Dis Colon Rectum 1998; 41: 93-7.

23. Ngo Y, Gelinet J, Ivanovic A, Kag J, Schenowitz G, Vilotte J, Rambaud J. Efficacy of a daily application of mesalazine (Pentasa) suppository with progressive release, in the treatment of ulcerative proctitis. A double-blind versus placebo randomized trial. Gastroenterol Clin Biol 1992; 16: 782-6.

24. Campieri M, Gionchetti P, Belluzzi A, Brignola C, Tampieri $\mathrm{M}$, Iannone $\mathrm{P}$, et al. Optimum dosage of 5-aminosalicylic acid as rectal enemas in patients with active ulcerative colitis. Gut 1991; 32: 929-31.

25. Williams C, Haber G, Aquino J. Double blind, placebocontrolled evaluation of 5-ASA suppositories in active proctitis and measurement of extent of spreads using 99m-Tc labelled 5-ASA suppositories. Dig Dis Sci 1987; 32: 71S-75S.

26. Van Bodegraven A, Boer B, Lourens J, Tuynmn H, Sindram J. Distribution of mesalazine enemas in active and quiescent ulcerative colitis. Aliment Pharmacol Ther 1996; 10: 327-32.

27. Campieri M, Paoluzi P, D’Albasio G, Brunetti G, Pera A, Barbara L. Better quality of therapy with 5-ASA colonic foam in patients with ulcerative colitis. Aliment Pharmacol Ther 1997; 11: 679-84.

28. Marteau P, Crand J, Foucault M, Rambaud J. Use of mesalazine slow release suppositories $1 \mathrm{~g}$ three times per week to maintain remission of ulcerative proctitis: a randomised double blind placebo controlled multicentre study. Gut 1998; 42: 195-9.

29. Mantzaris G, Hatzis A, Petraki K, Spiliadi C, Triantaphyl- 
lou G. Intermittent therapy with high-dose 5-aminosalicylic acid enemas maintains remission in ulcerative proctitis and proctosigmoiditis. Dis Colon Rectum 1994; 37: 58-62.

30. Banerjee S, Peppercorn M. Inflammatory bowel disease: medical therapy for specific clinical presentations. Gastroenterol Clin N Am 2002; 31: 185-202.

31. Regueiro M, Loftus E, Steinhart H, Cohen R. Medical management of left-sided ulcerative colitis and ulcerative proctitis: Clinical Evaluation of Therapeutic Trials. Inflamm Bowel Dis 2006; 12: 979-94.

32. Lakatos P, Lakatos L. Ulcerative proctitis: a review of pharmacotherapy and management. Expert Opin Pharmacother 2008; 9: 741-9.

33. Marshall J, Irvine E. Rectal corticosteroids versus alternative treatment in ulcerative colitis: a meta-analysis. Gut 1997; 40: 775-81.

34. Truelove S, Hambling M. Treatment of ulcerative colitis with local hydrocortisone hemisuccinate sodium; a report on a controlled therapeutic trial. BMJ 1958; 2: 1072-7.

35. Hanauer S, Robinson M, Pruitt R, Lazenby A, Persson T, Nilsson L, et al. Budesonide enema for treatment of active, distal ulcerative colitis and proctitis. A dose ranking study. U.S. Budesonide enema study group. Gastroenterology 1998; 115: 525-32.

36. Bar-Meir S, Fidder H, Faszczyk M, Bianchi G, Stuirnolo $\mathrm{G}$, Mickisch $\mathrm{O}$, et al. Budesonide foam $v$ s hidrocortisone acetate foam in the treatment of active ulcerative proctosgmoiditis. Dis Colon Rectum 2003; 46: 929-36.

37. Lobo A, Burke D, Sobala G, Axon A. Oral Tobramycin in ulcerative colitis: effect on maintenance of remission. Aliment Pharmacol Ther 1993; 7: 155-8.

38. Turunen U, Frakkila M, Hakala K, Seppala K, Sivonen A, Ogren $\mathrm{M}$, et al. Long-term treatment of ulcerative colitis with ciprofloxacin: a prospective double-blind, placebo-controlled study. Gastroenterology 1998; 115: 1072-8.

39. Ejderhamm J, Browaldh L, Oldaeus G, Saalman R, Stenhammar L. Treatment with glucocorticosteroid enemas in children with ulceratice colitis; a randomized singleblind multicenter comparison between budesonide and prednisolone. Gut 1999; 45 (Suppl V): A170.

40. Gisbert J, Gomollon F, Maté J, Pajares J. Preguntas y respuestas sobre el papel de la azatioprina y la 6-mercaptopurina en el tratamiento de la enfermedad inflamatoria intestinal. Gastroenterol Hepatol 2002; 25: 401-15.

41. Jewell D, Truelove S. Azathioprine in ulcerative colitis: final report on controlled therapeutic trial. BMJ 1974; 4: 627-30.

42. Caprilli R, Carratu R, Babbini M. A double-blind com- parison of the effectiveness of azathioprine and sulfasalzine in idiopathic proctocolitis. Preliminary report. Dig Dis 1975; 20: 115-20.

43. Sood A, Midha V, Sood N, Kaushal V. Role of azathioprine in severe ulcerative colitis: one-year, placebo- controlled, randomized trial. Indian J Gastroenterol 2000; 19: $14-6$.

44. Shibolet O, Regushevskaya E, Mayer Brezis, SoaresWeiser K. Cyclosporine A for induction of remission in severe ulcerative colitis (Review). Cochrane Database of Syst Rev 2005; 1: 1-16.

45. Breuer R, Soergel K, Lashner B, Christ M, Hanauer S, Vanaguna A, et al. Short-chain fatty acid rectal irrigation for left-sided ulcerative colitis: a randomised, placebo controlled trial. Gut 1997; 40: 485-91.

46. Hyams J, Davis P, Lerer T, Colletti R, Bousvaros A, Leichter A, et al. Clinical outcome of ulcerative proctitis in children. J Pediatr Gastroenterol Nutr 1997; 25: 149-52.

47. Hanauer S, Feagan B, Lichtenstein G, Mayer L, Schreiber $S$, Colombel J, et al. Mantainance infliximab for Crohn's disease: the ACCENT I randomized trial. Lancet 2002; 359: 1541-9.

48. Van Der Hagen S, Baeten C, Soeters P, Russel M, BeetsTan R, Van Gemert W. Anti-TNF-alpha (Infliximab) used as induction treatment in case of active proctitis in a multistep strategy followed by definitive surgery of complex anal fistulas in Crohn's disease: a preliminar report. Dis Colon Rectum 2005; 48: 758-67.

49. Sandborn W, Tremaine W, Leighton J, Lawson G, Zins B, Compton R, et al. Nicotine tartrate liquid enemas for midly and moderately active left-sided ulcerative colitis unresponsive to first-line therapy: a pilot study. Aliment Pharmacol Ther. 1997; 11: 661-71.

50. Bloom S, Kiilerich S, Lassen M, Forbes A, Leiper K, Langholz E, et al. Low molecular weight heparin (tinziparin) $v s$ placebo in the treatment of mild to moderately active ulcerative colitis. Aliment Pharmacol Ther 2004; 19: 871-8.

51. Bibiloni R, Fedorak R, Tannock G, Madsen K, Gionchetti $\mathrm{P}$, Campieri $\mathrm{M}$, et al. VSL\#3 probiotic-mixture induces remission in patients with active ulcerative colitis. Am J Gastroenterol 2005; 100: 1539-46.

52. Moum B, Ekbom A, Vatn M, Aadland E, Sauar J, Lygren I, et al. Clinical course during the 1st year after diagnosis in ulcerative colitis and Crohn's disease. Results of a large, prospective population-based study in Southeastern Norway, 1990-93. Scand J Gastroenterol 1997; 32: 1005-12.

53. Ritchie J, Powell-Tuck J, Lennard-Jones J. Clinical outcome of the first ten years of ulcerative colitis and proctitis. Lancet 1978; 1: 1140-3. 
54. Gionchetti P, Ardizzone S, Benvenuti M, Bianchi-Porro G, Biasco G, Cesari P, et al. A new mesalazine gel enema in the treatment of left-sided ulcerative colitis: a randomized controlled trial. Aliment Pharmacol Ther 1999; 13: 381-8.

55. Sinha A, Nightingale J, West K, Berlanga-Acosta J, Playford R. Epidermal growth factor enemas with oral mesalamine for mild-to-moderate left-sided ulcerative colitis or proctitis. N Engl J Med 2003; 349: 350-7.

56. Mariyama K, Takeshima F, Hamamoto T. Efficacy of
Rebamipide enemas in active distal ulcertive colitis and proctitis: A prospective study report. Dig Dig Sci 2005; 50: 2323-9.

57. Arakawa T, Kobayashi F, Yoshiwaka T, Tarnawski A. Rebamipide: overview of its mechanism of action and efficacy in mucosal protection and ulcer healing. Dig Dis Sci 1998; 43 (Suppl 9): 5S-13S.

58. Lawrence I, Copeland T. Rectal Tacrolimus in the treatment of resistant ulcerative proctitis. Alimen Pharmacol Ther 2008; 28: 1214-20. 